\section{Combination of negative pressure wound therapy and systemic steroid therapy in postsurgical pyoderma gangrenosum after reduction mammoplasty; a case of proven efficacy and safety}

\author{
Sara Tanini, Gianmarco Calugi, \\ Giulia Lo Russo \\ Plastic and Reconstructive \\ Microsurgery, Department of Surgery \\ and Translational Medicine, Careggi \\ University Hospital, Florence, Italy
}

\begin{abstract}
Pyoderma gangrenosum (PG) is a rare non-infective inflammatory disease of unknown etiology characterized by cutaneous papulo-pustoles that rapidly evolve into painful ulcerative lesions. Postoperative PG (PPG) is a clinical variant of $\mathrm{PG}$ in which pathergic response occurs at surgical sites. It is important to include PG in the differential diagnosis of breast ulceration. An incorrect diagnosis and treatment can potentially worsen the patient state, causing disfigurement with extensive scarring, an unpleasant aesthetic result and produce consequent psychological trauma.

We report a case of PPG after bilateral breast reduction mammoplasty treated with negative pressure wound therapy (NPWT) as local treatment for wound dehiscence in combination with systemic steroid therapy. This synergism led to a good aesthetic result. NPWT improved wound perfusion, it decreased the exudate, and promoted adherence of the mammary gland to the pectoral muscle. After 31 days deep sutures were placed to directly close the wound and the patient was discharged.

PPG is a rare, devastating occurrence after surgery. Surgeons must know that PPG is an evenience that can occur in otherwise healthy patients and must be considered in the differential diagnosis in case of necrotic ulcers and apparent infection status. NPWT resulted to have benefits in the treatment of PPG, with a role in limiting the size of the defect, avoiding pathergic self-reaction and allowing a faster recovery with higher chances of achieving a better result.
\end{abstract}

\section{Introduction}

Pyoderma gangrenosum (PG) is a rare destructive, non-infective inflammatory cutaneous disease of unknown etiology characterised by cutaneous papulo-pustoles that rapidly evolve into painful ulcerative lesions. ${ }^{1}$ It belongs to the spectrum of neutrophilic dermatoses caused by genetic predisposition and modified immune/inflammation response. ${ }^{2,3}$ It is usually associated with several diseases and has also been reported as a complication of many surgeries. ${ }^{1,2}$ Postoperative PG (PPG) is a clinical variant of $\mathrm{PG}$ in which pathergic response occurs at surgical sites. ${ }^{2,4}$ It is important to include $P G$ in the differential diagnosis of breast ulceration, because it is often initially diagnosed as a wound infection but, antibiotic therapy and wound debridement fail to arrest the rapid ulcer enlargement. ${ }^{5}$ PG insidiously causes a rapid and devastating soft tissue damage, the key points to avoid an excessive surgical demolition and obtain an acceptable outcome, are recognizing it quickly and setting an appropriate therapy in the least time possible; it can be especially important considering the aesthetic criteria in breast surgery. ${ }^{1,3}$ We report a case of PPG after breast reduction mammoplasty in a young woman who had no history of associated systemic disorder in which we obtained a good aesthetic result with a combination of negative wound therapy and systemic steroid therapy.

\section{Case Report}

A 39-year-old woman was admitted to our department requesting a bilateral breast reduction after massive weight loss (Figure 1). Past medical history was unremarkable and she underwent a previous gastric bypass as therapy to manage her severe obesity and a sequential abdominoplasty, without any complications. An inverted T-shaped reduction mammoplasty was performed in standard fashion removing $490 \mathrm{~g}$ of tissue from left breast and $520 \mathrm{~g}$ of tissue from right breast. The immediate postoperative course was uneventful. On postoperative day 4 , patient developed a fever of $38^{\circ} \mathrm{C}$, with severe pain (grade 7 of the visual ana$\log$ scale) and swelling of both breasts. Breasts were unsymmetrically involved, both presenting rapidly expanding necrotic wound areas, surrounded by an erythematous zone, with undermined borders. Postoperative infectious cellulitis was suspected and a blind antibiotic intravenous treatment was given to the patient waiting for the microbiological results and blood cultures. On postoperative day 7 fever was still present and the breast ulcer deteriorated by 1 to $2 \mathrm{~cm}$ per day with an excruciating
Correspondence: Sara Tanini, Plastic and Reconstructive Microsurgery, Department of Surgery and Translational Medicine, Careggi University Hospital, Florence, Italy.

E-mail: sarataninist@gmail.com

Key words: Negative-pressure wound therapy; vacuum therapy; pyoderma gangrenosum; post-operative complications; wound healing.

Received for publication: 3 May 2017. Accepted for publication: 11 October 2017.

This work is licensed under a Creative Commons Attribution-NonCommercial 4.0 International License (CC BY-NC 4.0).

(C) Copyright S. Tanini et al., 2017

Licensee PAGEPress, Italy

Dermatology Reports 2017; 9:7209

doi:10.4081/dr.2017.7209

pain (visual analog scale 9), and rapidly developing a $10 \times 5 \mathrm{~cm}$ of dimension cutaneous ulcer occupying the inferior half of the left breast but relatively sparing part of the areola and nipple. Along with the worsening of local conditions we assisted at a systemic drop with retro-nuchal pain, positive Lasegue's sign and hemianopia of the right eye. All these signs were orienting us towards a septic state. The microbiological cultures and the blood cultures for aerobic and anaerobic bacteria came back negative, so we proceeded to obtain tissue biopsies. The patient underwent partial surgical debridement and a local negative pressure wound therapy (125 $\mathrm{mmHg}$ ) was applied bilaterally. Two days after there was a new dehiscence in the surgical wound, with a large area of necrosis without secretion. Under analgesia the medications were changed.

Seven days after the onset of symptoms histological examination identified a nonspecific acute inflammatory process and ulceration with necrosis and neutrophilic infiltration into the dermis. A diagnosis of PPG was made and a systemic corticosteroid therapy was started. Patient was treated at the time of diagnosis with IV metilprednisone $(1.2 \mathrm{mg} / \mathrm{kg} / \mathrm{die})$ administration, according to international guidelines for PPG management, with a dramatic recovery. In the following days, the patient's condition improved, and we were able to observe granulation tissue, the contraction of wound edges and an initial adherence between tissues. NPWT was applied for one month, and thirty-five days after the initial procedure some stitches were applied to increase improvements of the wound's appearance (Figure 2). The corticosteroids dose was gradually reduced. 
The synergy of systemic steroid therapy and local negative pressure wound therapy led to an acceptable result, despite PSPG is a medical challenge. There has been a rapid response and wound areas significantly reduced. Wound bed showed an intensive vascularization, granulation tissue and a rapid contraction of wound edges, keeping the left nipple-areola complex vital and allowing a simple primary wound closure (Figure 3).

\section{Discussion}

Pyoderma gangrenosum is a rare dermatological disorder first described by Brocq in 1916. PG is an uncommon neutrophilic dermatosis that presents as an inflammatory and ulcerative disease of the skin, characterized by atypical signs, similar to infection disease. ${ }^{3,6} \mathrm{PG}$ is associated with a variety of inflammatory diseases (from $47.8 \%$ to $80 \%$ ), ${ }^{1}$ most commonly inflammatory bowel disease, rheumatoid arthritis, hematologic disease and malignancies. ${ }^{6}$ In up to $50 \%$ of cases, lesions occur at sites of cutaneous trauma, a phenomenon known as pathergy. These include sites of venipuncture, laparoscopy, and surgical incisions. Post-surgical pyoderma gangrenosum refers to the development of pyoderma gangrenosum at surgical sites in the immediate post-operative period. ${ }^{5}$ Clinical aspects often mimic a suppurative process. The initial signs of this condition are represented by erythema, wound tenderness and wound dehiscence that may occur from 4 to 14 days after the surgical procedure. These lesions rapidly evolve with a destructive, painful and progressive necrotic process that usually involves deep tissues. ${ }^{1,5-8}$

Although rare, PPG has been reported following plastic surgery to the breast. PG diagnosis after reductive mammoplasty is a challenge because clinical signs resemble an infectious process. Usually it is a diagnosis of exclusion based on clinical factors, ulcerative characteristics, negativity of microbiological culture and antibiotics treatment failure. ${ }^{1,9}$ Some clinical characteristics are usually associated with PPG: there is often a bilateral involvement, with a possible pathognomonic feature represented by the demarcated sparing of the nipple-areolar complex despite extensive ulceration of the surrounding skin. This striking observation may help in the diagnostic workup by excluding the other causes of necrosis such as flap failure, infection and necrotizing fasciitis in which the nipple-areolar complex is usually involved. ${ }^{6}$
Inefficacy of systemic wide spectrum antibiotic therapy, deterioration of patient's condition with a progressive wound enlargement often lead surgeons to aggressively debride the wound, worsening the pathergy of the disease. The surgical management of active PPG ulcers is discouraged due to the risk of pathergy-associated worsening. ${ }^{6,10}$ Early diagnosis and a correct therapy are the keystones to reduce the need

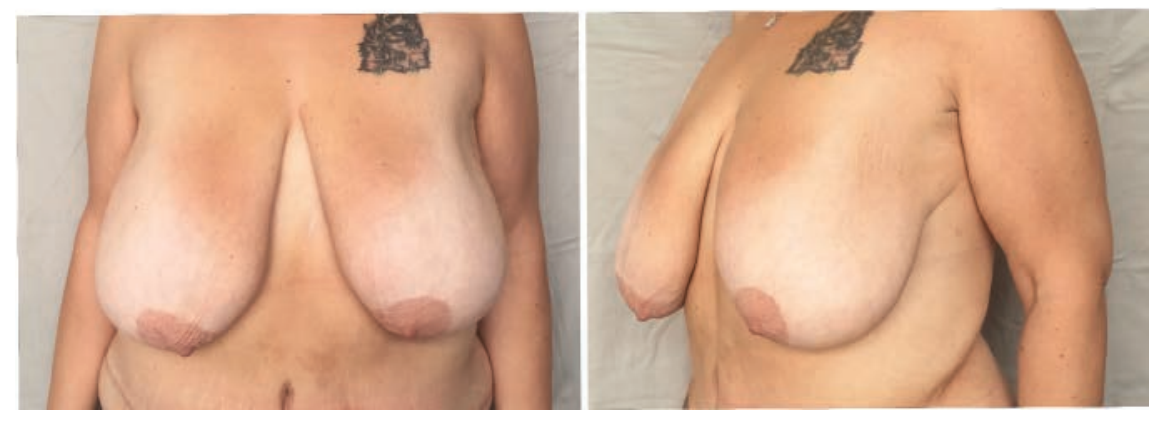

Figure 1. Frontal and lateral preoperative view.
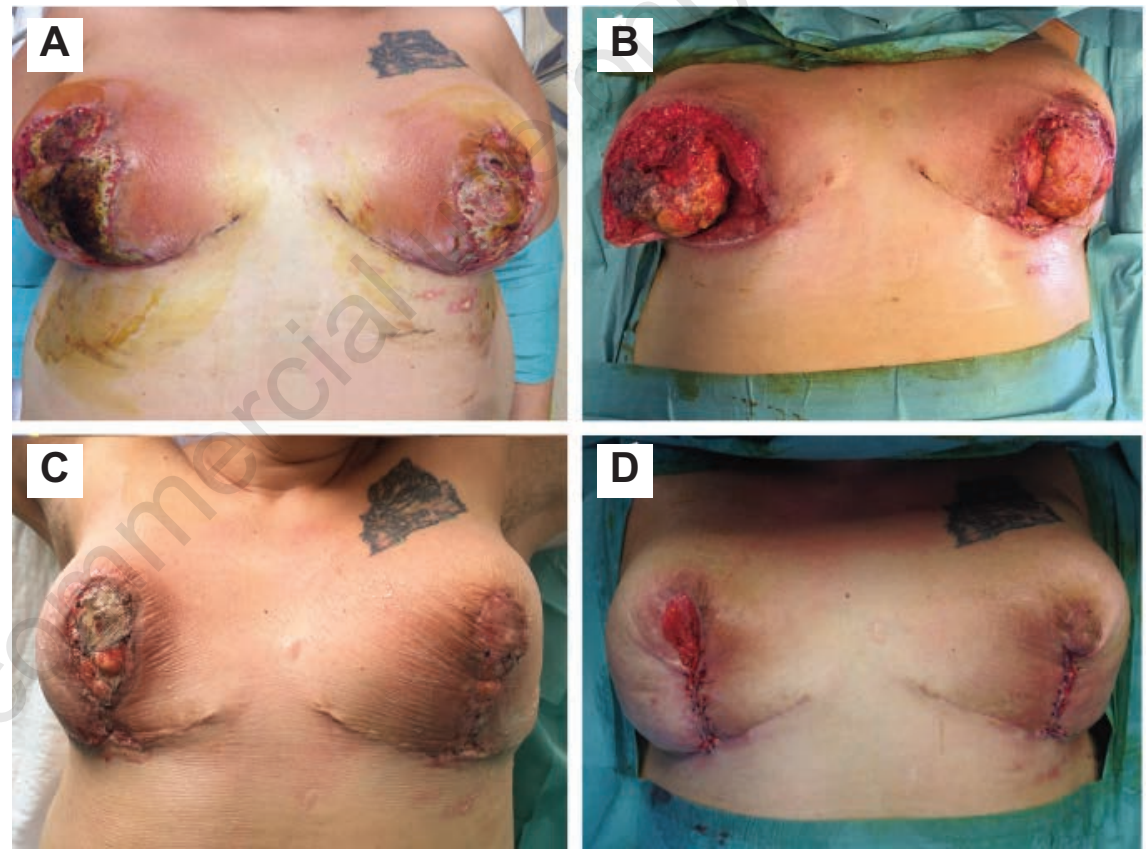

Figure 2. A) Six days after bilateral breast reduction mammoplasty. B) Nine days postoperatively. Increased area of necrosis and progressive aggravation of wound areas. The patient underwent partial surgical debridement and a local negative pressure wound therapy was applied bilaterally. C) Twenty days after initial procedure. Improved appearance of the wound after 3 foam changes. D) Thirty-five days after initial procedure. To increase the improvement of wound's appearance some stitches were applied.
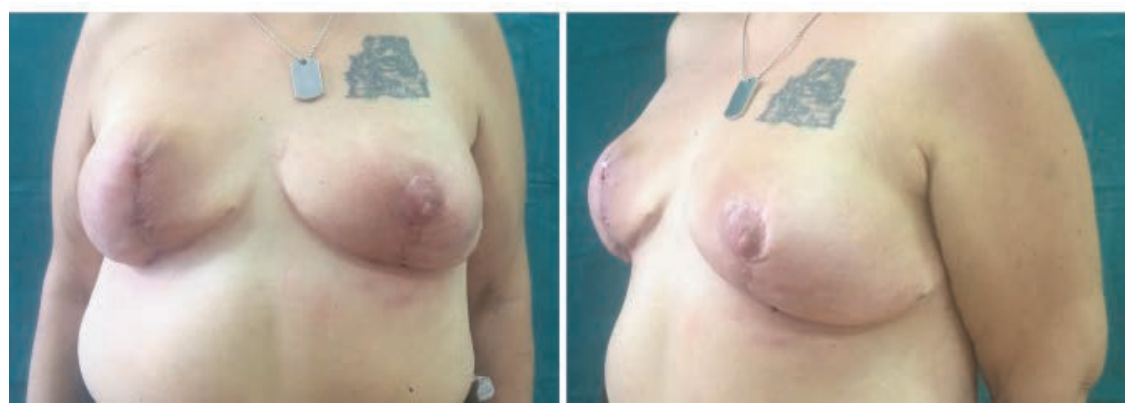

Figure 3. Two months post op follow-up frontal and lateral view. 
of secondary surgical procedures that lead to a good aesthetic result. ${ }^{6}$

The goal of treatment is to limit tissue destruction and promote wound healing.,11 Even though there is no specific treatment for $P P G,{ }^{12,13}$ immunomodulatory and immunosuppressive systemic therapy are the mainstay for the treatment of PG ulcers. Corticosteroids in moderate to high doses (prednisolone, $0.5-2 \mathrm{mg} / \mathrm{kg} /$ day) and cyclosporine (3-6 mg/kg/day) are the most frequently used drugs, and also the best documented in literature. ${ }^{14}$ In 1997, Argenta and Moryskwas, developed a commercial product based on the use of negative pressure and began to use it for wound treatment, providing an excellent environment for faster healing. ${ }^{11}$

The rational of the use of sub atmospheric pressure therapy includes: i) increased tissue perfusion; ${ }^{10}$ ii) control of the advancing ischemic process; iii) rapid production of a healthy granular wound bed; iv) reduced bacterial load that provides local control of the moisture, exudates and edema besides achieving an important pain relief. ${ }^{15}$

NPWT may have benefits in the treatment of complicated breast incisions and injuries. In literature there are descriptions of the use of NPWT after breast surgery such as autologus brest reconstrucion, additive mastoplasty, mastectomy, TRAM flap necrosis and tissue expander infection. No complications with the use of NPWT have been reported. ${ }^{16}$

In our case we had a patient with a massive loss of mammary tissue bilaterally. On one side we had the need to protect the substance loss from infection and reduce the expansion of the ulcer, while on the other side we needed to support the healing process and obtain the best result possible. These reasons lead us to a combined treatment of negative wound pressure therapy and systemic steroid therapy.

In our experience, the use of negative pressure wound therapy improved wound perfusion, decreased exudate, and promoted adherence of the mammary gland to the pectoral muscle. This led to an effective local disease control, avoiding further need of surgical escharectomy but most importantly, it permitted faster healing of the very large ulcers of the patient, reducing significantly wound dehiscence, with a complete resolution of the erythematous undermined borders. Despite patient's pain, VAC therapy is well-tolerated. ${ }^{13}$
The time needed for complete healing after PPG is described in the literature as ranging from 1.5 months to 1 year (average, 5 months). After the third VAC foam change, it was possible to place fastening sutures between these tissues. Clinical follow-up showed good results and a reduced length of hospital stay according to literature. ${ }^{11}$

Use of NPWT for breast wounds might be benefical as it is for other wounds. However, large prospective controlled studies that compare standard treatment with the use of NPWT are needed to evaluate the main role of NPWT. ${ }^{16}$

\section{Conclusions}

PPG is a rare, devastating occurrence after surgery. A conservative treatment was the key to success. Surgeons must know that PPG is an evenience that can occur in otherwise healthy patients and must be considered in the differential diagnosis in case of necrotic ulcers and apparent infection status. In our case, the early recognition and application of NPWT in combination with systemic steroid therapy has limited the size of the defect, avoiding pathergic self-reaction and allowing a faster recovery with higher chances of achieving a better result.

\section{References}

1. Abtahi-naeini B, Bagheri F, Pourazizi $M$, et al. Unusual cause of breast wound: postoperative pyoderma gangrenosum. Int Wound J 2017;14:285-7.

2. Larcher L, Schwaiger K, Eisendle K, et al. Aesthetic breast augmentation mastopexy followed by postsurgical pyoderma gangrenosum (PSPG): clinic, treatment, and review of the literature. Aesth Plast Surg 2015;39:506-13.

3. Marinopoulos S, Theofanakis C, Zacharouli $\mathrm{T}$, et al. Pyoderma gangrenosum of the breast: a case report study. Int J of Surg Case Rep 2017; 31:203-5.

4. Tolkachjov SN, Fahy AS, Wetter DA, et al. Postoperative pyoderma gangrenosum (PG): the Mayo Clinic experience of 20 years from 1994 through 2014. J Am Acad Dermatol 2015;73:615-22. 5. Zuo KJ, Fung E, Tredget EE, Lin AN. A systematic review of post-surgical pyoderma gangrenosum: identification of risk factors and proposed management strategy. J Plast Reconstr Aesthet Surg 2015;68:295-303.

6. Su WP, Davis MD, Weening RH, et al. Pyoderma gangrenosum; clinicopathologic correlation and proposed diagnostic criteria Int J Dermatol 2004;43:790800.

7. Goshtasby PH, Chami RG, Michael R. A novel approach to the management of pyoderma gangrenosum complicating reduction mammoplasty. Aesthet Surg J 2010;30:186-94.

8. Gameiro A, Pereira N, Cardoso JC, Gonçalo M. Pyoderma gangrenosum: challenges and solutions Clin Cosmet Investig Dermatol 2015;28:285-93.

9. Duval A, Boissel N, Servant JM, et al. Pyoderma gangrnosum of the breast: a diagnosis not to be missed. J Plast Reconstr Aesthet Surg 2011;64:17-20.

10. Ghersi MM, Ricotti C, Nousari CH, Newman MI. Negative pressure dressing in the management of pyoderma gangrenosum ulcer. Arch Dermatol 2007;143:1249-51.

11. Soncini JA, Grassi Salles A, Frizzo Neto JA, Gemperli R. Successful treatment of pyoderma gangrenous after augmentation mastopexy using vacuum therapy. Plast Reconstr Surg Glob Open 2016;9:e1072.

12. Schintler MV, Grohmann M, Donia C, et al. Management of an unfortunate triad after breast reconstruction: pyoderma gangrenosum, fullthickness chest wall defect and Acinetobacter baumannii infection. J Plast Reconstr Aesthet Surg 2012;63: 564-7.

13. Callen JP. Pyoderma gangrenosum and related disordes. Dermatol Clin 1990; 7:1249.

14. Chow RKP, Ho VC. Treatment of pyoderma gangrenosum J Am Acad Dermatol 1996;34:1047.

15. Fraccalvieri M, Fierro MT, Salomone $\mathrm{M}$, et al. Gauze-based negative pressure wound therapy: a valid method to manage pyoderma gangrenosum. Int Wound J 2014;11:164-8.

16. Namdaroğlu OB, Yazıcı H, Müctebaztürk A, et al. Negative-pressure wound therapy in chronic inflammatory breast diseases. J Breast Health 2016;12:91-3. 